\title{
Aerosolization of Mycobacterium tuberculosis by tidal breathing
}

(Short title: Tidal breathing generates tuberculosis bioaerosols)

Ryan Dinkele, ${ }^{1,2}$ Sophia Gessner, ${ }^{1,2}$ Andrea McKerry, ${ }^{3}$ Bryan Leonard, ${ }^{3}$ Juane Leukes, ${ }^{3}$ Ronnett Seldon, ${ }^{3}$ Digby F. Warner, ${ }^{1,2,4^{*}}$ and Robin Wood ${ }^{2,3^{*}}$

1SAMRC/NHLS/UCT Molecular Mycobacteriology Research Unit \& DST/NRF Centre of Excellence for Biomedical TB Research, Department of Pathology, Faculty of Health Sciences, University of Cape Town, South Africa.

${ }^{2}$ Institute of Infectious Disease and Molecular Medicine, Faculty of Health Sciences, University of Cape Town, South Africa.

${ }^{3}$ Desmond Tutu Health Foundation, University of Cape Town, South Africa.

${ }^{4}$ Wellcome Centre for Infectious Disease Research in Africa, Faculty of Health Sciences, University of Cape Town, South Africa.

${ }^{\star}$ Corresponding authors:

Robin Wood: robin.wood@hiv-research.org.za, +27 21650 6966; Digby Warner: digby.warner@uct.ac.za,+27 214066556. 


\section{Abstract}

2 Rationale: Interrupting tuberculosis (TB) transmission requires an improved 3 understanding of how - and when - the causative organism, Mycobacterium 4 tuberculosis (Mtb), is aerosolized. Although Cough is commonly assumed to be the 5 dominant source of Mtb aerosols, recent evidence of Cough-independent Mtb release 6 implies the contribution of alternative mechanisms.

7 Objective: To compare the aerosolization of Mtb and particulate matter from 8 GeneXpert-positive patients during three separate respiratory manoeuvres: Tidal 9 Breathing (TiBr), Forced Vital Capacity (FVC), and Cough.

Methodology: Bioaerosol sampling and Mtb detection were combined with real-time assessments of $\mathrm{CO}_{2}$ production and particle counts from 39 confirmed TB patients.

Measurements and Main Results: TiBr and FVC produced comparable numbers of particles, with Cough producing $>4$-fold more. For all manoeuvres, the proportions of particles detected across size categories from $0.5-5 \mu \mathrm{m}$ were similar, with minor differences observed only in particles between $1.5-2 \mu \mathrm{m}(\mathrm{p}=0.014)$ and $>5 \mu \mathrm{m}(\mathrm{p}=$ $0.020)$. Viable Mtb bacilli were detected in $66 \%, 70 \%$, and $65 \%$ of $\mathrm{TiBr}, \mathrm{FVC}$, and Cough samples, respectively. Notably, while Cough produced 3-fold more Mtb than $\mathrm{TiBr}$, the relative infrequency of coughing compared to breathing implies that TiBr likely contributes $>90 \%$ of the daily aerosolised Mtb across a range of Cough frequencies.

Conclusions: Our results suggest that, while Cough increases particle aerosolization compared to $\mathrm{TiBr}$, this is not associated with increased Mtb aerosolization. Instead, TiBr produces more Mtb per particle than Cough. Assuming the number of viable Mtb organisms detected provides a proxy measure of patient infectiousness, these observations imply a significant contribution of $\mathrm{TiBr}$ to $\mathrm{TB}$ transmission.

Key words: TB transmission, bioaerosol, cough, forced vital capacity. 
Introduction

Chronic Cough is a hallmark symptom of tuberculosis (TB), an airborne infectious disease which is caused by Mycobacterium tuberculosis (Mtb) and is associated with high global mortality and morbidity (1). Given its importance in TB diagnosis, Cough has unsurprisingly been central to TB transmission research (2). There are multiple lines of evidence, however, which suggest that the focus on Cough risks ignoring other important contributing mechanisms, undermining the implementation of new approaches to reducing TB transmission - especially in TB endemic settings. For example, a recent national TB prevalence survey in South Africa (consistently among the WHO's annual list of high TB burden countries) reported that nearly $60 \%$ of individuals with bacteriologically confirmed pulmonary TB were asymptomatic (3). Similarly, a pioneering face-mask sampling study noted no association between Cough frequency and the detection of Mtb organisms (4). When considered with modelling data which estimate 1.7 billion latent Mtb infections globally (5), these observations imply that most Mtb infections are not associated with Cough as pathognomonic symptom - even when Mtb bacilli are present at detectable levels (3). Given the low proportion of infections leading to symptomatic disease and the scale of the TB epidemic, it is conceivable that Mtb transmission from symptomatic individuals, and therefore Cough, is highly efficient. However, considering the challenges in identifying TB transmitters (6) and the lack of association between Mtb detection and Cough frequency (4), an alternative must be considered: is TB transmission primarily accomplished by Cough-independent means?

We have developed a platform combining non-invasive bioaerosol capture technology and fluorescence microscopy to enumerate viable Mtb released by confirmed TB patients (7-9). Using this platform, we detected Mtb bacilli in the absence of (induced) Cough. More recently, we compared deep exhalations to Cough and found no difference in Mtb aerosolization (10). However, these observations were not conclusive given that we did not directly investigate the propensity for respiratory aerosol production in each manoeuvre. Therefore, we aimed in the current work to investigate the potential for $M t b$ release via Tidal Breathing ( $\mathrm{TiBr}$ ), Forced Vital Capacity (FVC) and Cough. As detailed below, our observations suggest that release 
62 of Mtb aerosols via $\mathrm{TiBr}$ might constitute an important contributor to ongoing transmission in both active TB disease and asymptomatic Mtb infection.

\section{Methods and materials}

\section{Participant recruitment}

Participants over 13 years of age returning a GeneXpert-positive sputum result were recruited from March 2020 to June 2021 at primary healthcare facilities in Ocean View and Masiphumelele, peri-urban townships in Cape Town, South Africa. Recruitment and sampling occurred prior to the initiation of standard anti-TB chemotherapy. Ethical approval was obtained from the Human Research Ethics Committee of the University of Cape Town (HREC 529/2019).

\section{Sample collection}

Bioaerosols from three respiratory manoeuvres, Forced Vital Capacity (FVC), Tidal Breathing ( $\mathrm{TiBr}$ ), and Cough, were captured in liquid cyclone collectors utilising a direct sampling strategy (Figure 1A) (10). A unidirectional airflow forced exhaled air via a $\mathrm{CO}_{2}$ monitor and a high-flow cyclone collector at a maximum flow rate of $300 \mathrm{~L} /$ minute, trapping particulate matter in the collection medium (sterilised phosphate-buffered saline, PBS). During $\mathrm{TiBr}$ sampling, each participant placed their head within the elliptical cone and breathed normally for five minutes. Bioaerosols were captured within the cyclone collector at $200 \mathrm{~L} /$ minute, while $100 \mathrm{~L} /$ minute of exhaled air was diverted to an aerodynamic particle sizer (APS). During FVC and Cough sampling, each participant performed 15 manoeuvres by placing their head in the elliptical cone every 15 seconds. Bioaerosols were captured within the cyclone collector at 300 $\mathrm{L} /$ minute for the first and last five manoeuvres. During the middle five manoeuvres, $100 \mathrm{~L} /$ minute of exhaled air was diverted via the APS. New cyclone collectors were attached after each sample allowing for the independent enumeration of Mtb utilising our previously described concentration and visualisation pipeline (8).

\section{Staining and visualisation of bioaerosol samples}

Bioaerosols were stained with DMN-trehalose (DMN-tre) (Olilux Biosciences Inc.) and visualised as previously outlined (8). Briefly, liquid-captured bioaerosols (5 - $10 \mathrm{~mL})$ were centrifuged for 10 minutes at $3000 \times g$ (Allegra X-15R, Beckman Coulter) and resuspended in $200 \mu \mathrm{L}$ of Middlebrook $7 \mathrm{H} 9$ medium supplemented with $100 \mu \mathrm{M}$ DMN- 
tre. Staining was done overnight, after which samples were concentrated at 13,000 $x$ $g$ for five minutes and resuspended in $20 \mu \mathrm{L}$ filtered PBS. Stained samples were loaded on nanowell-arrayed microscope slides and viewed on a Zeiss Axio Observer 7 with widefield illumination from a $475 \mathrm{~nm}$ LED and a Zeiss 38 HE filter set. A 100x plan-apochromatic phase 3 oil immersion objective $(\mathrm{NA}=1.4)$ was used.

\section{Statistical methods}

Each participant performed three respiratory manoeuvres violating the assumption of independence. Therefore, to account for this correlation within the data, various Linear Mixed Models were used as the incorporation of the random effect enabled the average differences between manoeuvres to be determined while accounting for variation between participants. For continuous outcomes, a $\log _{10}$-transformation was performed and linearity, normality of residuals, and homoskedasticity assessed. For binary outcomes, logistic regression was performed with sample type as the fixed effect, and variation in slope (random effects) accounted for by participant. For count data, negative binomial regression was applied. Detailed statistical and data wrangling methods can be found in the online supplement. Data were analysed in R studio (11) with $\mathrm{R}$ version 4.0 .3 (12).

\section{Results}

Detection and quantification of particle release during different respiratory manoeuvres

Direct bioaerosol sampling was performed on 39 participants with corresponding $\mathrm{CO}_{2}$ and particle data obtained for 32 and 33 participants, respectively; this ensured a final sample size of 32 (Figure E1). FVC and Cough samples were excluded if fewer than two peaks in particle counts were detected above background. Owing to variations in sampling duration, samples were assessed in three ways (Figure 1B): the total number or volume of particles collected, the average number or volume of particles produced per manoeuvre, and the estimated total number or volume of particles produced.

During the particle sampling window, similar numbers (Figure 2A) and volumes (Figure E2A) of particles were collected for $\mathrm{TiBr}$ and Cough, with FVC producing significantly fewer particles than $\mathrm{TiBr}$. However, after averaging the number of particles per manoeuvre, it was clear that $\mathrm{TiBr}$ produced a significantly lower number 
124 (Figure 2B) and volume (Figure E2B) of particles compared to either FVC or Cough.

125 Interestingly, when considering the total number of manoeuvres performed, $\mathrm{TiBr}$ and

126 FVC produced comparable numbers (Figure 2C) and volumes (Figure E2C) of

127 particles, with Cough producing more than 4-fold more particles than $\mathrm{TiBr}$. Inter-

128 participant variability contributed approximately $12 \%$ of the total variation when 129 examining particle numbers per manoeuvre (Figure 2B), and approximately 16\% of

130 the variation when examining particle volumes per manoeuvre (Figure E2B). Together,

131 these data suggest that variation in particle production between the three respiratory

132 manoeuvres contributed to variation in the overall volume of bioaerosol collected after

1335 minutes of sampling; however, no manoeuvre was significantly under-sampled.

Size stratification of particles enables more specific comparisons of respiratory manoeuvres

136 Particles of various sizes are aerosolised and may differ between respiratory 137 manoeuvres. The APS binned particles into size categories (Figure 3A); therefore, we 138 examined the effect of each manoeuvre on the distributions of particles across categories. The average number (Figure 3B) and volume (Figure E3A) of particles per manoeuvre were stratified by size category and sample type, with the individual data from each participant overlayed. Two features were apparent: firstly, there was a consistent distribution in average count per size category across all three manoeuvres. Moreover, this trend was recapitulated when the proportion of each size category relative to the total particle count per manoeuvre was compared for each size category (Figure 3C). Only minor variations were detected, with levels of significance reached solely for C.3 $(1.5-2 \mu \mathrm{m})$ and C.5 $(>5 \mu \mathrm{m})$. This suggested that factors leading to increased bioaerosol generation did not affect the distribution of size categories aerosolised over the size range measured. The second striking observation was the per patient consistency in the relationship between the different size categories. It was also apparent that size category was not a confounder of the relationship between the manoeuvre type and particle count per manoeuvre (Figure E3B), as the average differences between $\mathrm{TiBr}$ and $\mathrm{FVC}$ and $\mathrm{TiBr}$ and closely recapitulated those observed in the previous model (Figure 2B). In combination, these results indicated that intrinsic differences in the propensity for total particle production separate individuals, and that these are conserved across particle sizes. 
Use of three independent cyclone collectors to enable enumeration of Mtb bacilli

The relative contributions of different respiratory manoeuvres to aerosolization of Mtb bacilli has been poorly studied, with most reports focusing on Cough. We implemented a sampling strategy comprising 15 FVC and Cough manoeuvres and five minutes of $\mathrm{TiBr}$. This resulted in closely matching volumes of bioaerosol collected for $\mathrm{TiBr}$ and FVC samples, with more than 3-fold the volume collected during Cough sampling (Figure E2C). Given the increased volume of bioaerosol collected from Cough and its assumed importance in TB transmission, we expected to find the greatest numbers of Mtb bacilli in the Cough samples.

The rate of production of $M t b$ was estimated for the three manoeuvres during the five minutes of sampling. For both FVC (IRR $=0.53, p=0.0971)$ and Cough (IRR $=0.51, p=0.0662)$, there was a trend to $M t b$ production at a lower rate compared to $\mathrm{TiBr}$; however, neither of these was statistically significant (Figure 4A). Surprisingly, the percent of positive samples was consistent for all three manoeuvres, with $66 \%$, $70 \%$, and $65 \%$ of the samples positive for Mtb in TiBr, FVC, and Cough, respectively. Additionally, no significant differences were detected in the odds ratio of detecting Mtb between $\mathrm{TiBr}$ and $\mathrm{FVC}$ or $\mathrm{TiBr}$ and Cough (Figure 4B).

The occurrence of spontaneous coughs during $\mathrm{TiBr}$ sampling might confound the $\mathrm{TiBr}$ measurement. To test this possibility, peaks detected in $\mathrm{TiBr}$ that were greater than 1.5 times the average peak height in the corresponding Cough sample were assumed to be spontaneous coughs (Figure E4A, right panel). Notably, plotting Mtb counts from $\mathrm{TiBr}$ against the number of coughs detected (Figure E4B) indicated no association between the number of $M t b$ and spontaneous coughs during sampling.

To examine the relationship between particle numbers and the aerosolization of $M t b$ bacilli, the relative abundance of $M t b$ bacilli per particle was calculated for all three manoeuvres. Participants with a zero count for Mtb were excluded from this analysis. The average concentration of $M t b$ bacilli for $\mathrm{TiBr}$ was $70 \%$ and $90 \%$ higher than that of FVC and Cough, respectively (Figure 5A). In addition, no correlation between total Mtb count and total particle count was observed for either FVC or Cough (Figure 5B). A slightly more apparent linear relationship was observed for $\mathrm{TiBr}$ (Figure 5B); however, this did not reach statistical significance. Together, these data imply a disconnect between the aerosolization of particles and Mtb. 
The extent of aerosolization of Mtb depends predominantly on manoeuvre frequency

Knowing the concentration of Mtb per volume of bioaerosol suggested the potential to gain useful insight into the relative contributions of Cough and $\mathrm{TiBr}$ to the daily production of $M t b$. To this end, we first compared the average number of bacilli produced per manoeuvre. On average, TiBr produced 2.6- and 3.2-fold fewer Mtb per manoeuvre compared to FVC and Cough, respectively (Figure 6A). Next, we extrapolated the values for the average number of bacilli per manoeuvre and the average frequency of manoeuvres per day to estimate daily Mtb production. Because FVCs are artificial, directed manoeuvres that are performed only under specific instruction, we utilized $\mathrm{TiBr}$ and Cough for this calculation.

Our data revealed that participant $\mathrm{TiBr}$ occurred at a rate of around one breath every 3.9 seconds, suggesting approximately 22,047 tidal breaths over 24 hours. Since we did not directly measure the frequency of spontaneous coughs, we estimated 24-hour Cough frequencies from published data (4), with a median of 466 coughs/day (first quartile 234, last quartile 551). We then used a constant maximum number of breaths per day $(22,047)$ and assumed that, for each Cough, there would be one fewer breath. The average number of Mtb bacilli produced by $\mathrm{TiBr}$ and Cough was then calculated, and the relative proportion determined by dividing the number per manoeuvre by the total. This enabled an estimation of the relative contribution per day for an average person with an increasing number of coughs (Figure 6B).

It was apparent that Cough contributed between 3 and $7 \%$ of the total number of $M t b$ bacilli released, with $\mathrm{TiBr}$ consistently producing over $93 \%$. Together, these data suggest that coughing is likely to produce significantly fewer bacilli per day compared to $\mathrm{TiBr}$. That is, while the higher per event number and velocity of bacillary release might ensure an important role for coughing in disease transmission in short contacts, for typical exposures in high-risk settings such as public transport, workplaces, schools, etc. (13), $\mathrm{TiBr}$ is expected to contribute significantly to $\mathrm{TB}$ prevalence, especially in high-burden settings such as South Africa.

\section{Discussion}

Cough has traditionally been considered the primary means of TB transmission (14). The result is that TB transmission research has predominately focused on factors 
221 including Cough production, frequency, and the Cough-borne Mtb bacillary load (2). However, the absence in all studies of a comparator respiratory manoeuvre (15-17)

223 has rendered impossible any assessment of alternative contributory mechanisms.

224 Transmission by aerosol requires the aerosolization of particles from the site of infection (18). For Mtb, which infects the peripheral lung and alveolar spaces (19), the proposed mechanism of particle aerosolization is fluid film rupture (20). According to this model, particles are produced during inspiration by alveolar reopening and released through expiration (21). Factors impacting particle release are therefore the rate of inspiration and the depth of expiration (21), with a recent study comparing deep exhalation and Cough finding no significant difference in the number of Mtb aerosolized between the two manoeuvres (10). For these reasons, we hypothesized that $\mathrm{TiBr}$ contributes to the aerosolization of $M t b$. Therefore, we sought in this study to directly compare the propensity for particle and Mtb aerosolization via three defined respiratory manoeuvres: Cough, FVC and $\mathrm{TiBr}$.

We sampled bioaerosols from 39 TB-positive participants. Consistent with findings from similar studies, $88 \%$ of participants produced at least one bioaerosol sample that was positive for $M t b(4,8,10)$, a marked increase over culture-based Cough-sampling techniques (17). Our results also indicated that all three respiratory manoeuvres were equally likely to produce $M t b$, with $\mathrm{TiBr}, \mathrm{FVC}$, and Cough returning positive signals in $66 \%, 70 \%$, and $65 \%$ of samples, respectively. When extrapolating based on daily manoeuvre frequency, these observations imply that $\mathrm{TiBr}$ contributes more than $90 \%$ of the daily aerosolised Mtb across a range of Cough frequencies - a conclusion consistent with the lack of correlation between Mtb aerosolization and Cough frequency (4).

Establishing a sampling algorithm appropriate for three distinct respiratory manoeuvres is challenging. However, the total number of particles produced during FVC and TiBr sampling were similar, with the Cough producing approximately 4-fold more particles. This suggested that, despite differences in sampling algorithms, the risk of under-sampling any manoeuvre was low. In addition, we saw significant variation between participants, spanning two orders of magnitude, consistent with previous observations (22). Per manoeuvre, TiBr produced significantly fewer particles than both FVC and Cough, with Cough producing the most particles. While it is 
increased number of particles produced by Cough, this interpretation seems unlikely given the similarity in particle counts for Cough and FVC. Considering Cough and FVC are quite different in the rate of expiration, it might be more instructive that both these manoeuvres require deep inspiration: the inference, then, that the rate of expiration and, therefore, the turbulence of expired air - plays a minimal role in aerosol generation is consistent with a fluid-film rupture model of aerosol generation in the peripheral lung (21). This is also consistent with the similarities in size distributions of particles between both participants and respiratory manoeuvres. Although the absolute counts per category varied between manoeuvres, the proportional compositions within each size category were conserved - an observation which supports the inference that the mechanism of particle production is consistent across the three respiratory manoeuvres $(21,22)$.

The average total number of Mtb per participant was 12.6 ( $\max =52$ ), consistent with our previous study (8). However, owing to continued enhancements of our bioaerosol collection system, participants were sampled for $\sim 15$ minutes in this study versus the 60-minute sampling duration reported previously (8). Unexpectedly, all three respiratory manoeuvres produced consistently low levels of $M t b$, with a mean count of $3.9(\max =21), 5.9(\max =39)$ and $3.4(\max =15)$ for FVC, TiBr and Cough, respectively. TiBr samples tended to have a two-fold higher rate of Mtb aerosolization compared to both FVC and Cough; however, these differences were not significant. And, as noted above, the probability of a sample returning a positive result was consistent for all respiratory manoeuvres. Notably, among the participants who generated at least one positive sample, most (27/28) produced Mtb within their FVC and/or TiBr sample. These findings suggest that induced Cough may be unnecessary in studying Mtb transmission, a potentially important innovation given the strenuous nature of the induced Cough especially for unwell patients.

Contrary to our expectations, the concentration of Mtb bacilli per particle was $70 \%$ or $90 \%$ lower in FVC and Cough, respectively, compared to $\mathrm{TiBr}$. In addition, no correlation was observed between particle number and Mtb count, even when stratified by participant. Together, these data suggest that variation in particle production alone is insufficient evidence to identify infectious patients, and that applications to reduce particle production seem unlikely to reduce infectiousness (23). 
Despite the apparent unlinking of particle count and Mtb aerosolization, the sizeable increase in aerosol production during FVC and Cough manifest as a 3-fold increase in Mtb aerosolization for these manoeuvres compared to $\mathrm{TiBr}$. However, when extrapolated to daily estimates, the relatively high frequency of breathing relative to coughing suggests that, over time, TiBr represents a major source of Mtb aerosols, as suggested previously (20). We calculated that, during any single day, breathing contributes $>90 \%$ of the $M t b$ aerosolised by a TB-positive individual.

Our study had a number of limitations. The sample collection algorithm was not consistent for all respiratory manoeuvres - $\mathrm{TiBr}$ samples were primarily defined by time versus FVC and Cough that were defined by event number - and the particle collection and measurement apparatus were connected in parallel and not in series. Consequently, extrapolations were required to estimate the total number of particles and organisms present in the entire bioaerosol. Additionally, no work was done to determine the effect of manoeuvre order on particle or Mtb production. This could have impacted particle and Mtb production through participant exhaustion or through particle clearance. That said, we did separate FVC and Cough samples to ensure that $\mathrm{TiBr}$ provided a rest period between strenuous samples. The participants included in this study were diagnosed TB-positive via GeneXpert. Therefore, we cannot conclude the relative importance of $\mathrm{TiBr}$ to asymptomatic transmission. While our data indicate that significant levels of Mtb are aerosolised daily independent of participant Cough, further work is required to investigate this hypothesis in GeneXpert-negative, asymptomatic individuals. Owing to technical challenges inherent in studying spontaneous Cough over short sampling periods, we only studied induced Cough which may not be as infectious. Nevertheless, spontaneous coughs were detected in several $\mathrm{TiBr}$ samples, with no effect on overall production of Mtb. In estimating Cough frequency per hour, we assumed that the rate is consistent throughout the day. This is a strong assumption, and it is more likely that coughs cluster into discrete events with multiple coughs occurring at a time, suggesting potential outbursts of infectious aerosol production. A final limitation is that, while it might reasonably be assumed that Mtb bioaerosol counts are directly linked to infectiousness, this has not been formally demonstrated. be a significant contributor to Mtb transmission in an endemic TB setting. This has 
319 significant ramifications for both transmission studies and intervention strategies.

320 Firstly, bioaerosol sampling lends itself to a non-invasive participant sampling.

321 Although the impact of induced Cough on a participant is relatively low, if a less

322 invasive sampling algorithm can be applied, it should be. Secondly, interventions

323 targeting disease transmission, such as active screening for symptomatic individuals,

324 may not be effective. Therefore, linking bioaerosol organisms with infectious potential

325 is of vital importance. Bioaerosol sampling is non-invasive and provides potential to

326 identify infectious individuals well in advance of any typical screening regimen. This

327 may offer a novel means to identify and treat infectious individuals before they

328 manifest with definite symptoms.

329 A paradigm in which Cough is the primary driver of TB transmission places 330 surpassing importance on lung pathology; moreover, it appears inconsistent with key

331 epidemiological observations. Sub-clinical TB infections could represent a novel and

332 uncontrolled source of disease transmission. Consequently, understanding how Mtb

333 bacilli are aerosolised is of critical importance to curbing the epidemic in high-burden

334 settings

336 Author contribution:

337 Conceptualization and design: RD, SG, AM, BL, JL, RS, DFW \& RW; acquisition of 338 data: JL, AM, BL, RS \& RW; analysis and interpretation: RD, DFW \& RW; first 339 manuscript draft: RD \& DFW; funding acquisition: DFW \& RW. All authors critically 340 reviewed and revised the manuscript for intellectual content and approved it prior to 341 submission.

\section{Acknowledgements}

343 We acknowledge the support of the South African Medical Research Council 344 (SAMRC) with funds from National Treasury under its Economic Competitiveness and 345 Support Package (MRC-RFA-UFSP-01-2013/CCAMP, RW), and the Strategic Health 346 Innovations Partnerships (SHIP) Unit of the SAMRC (DFW) and as a sub-grant from 347 the Bill and Melinda Gates Foundation (RW). We are grateful to the Bill \& Melinda 348 Gates Foundation (OPP1116641, RW), the US National Institutes of Health (NIH 349 R37Al058736, Freedberg PI; RW), the Research Council of Norway (R\&D Project 
bioRxiv preprint doi: https://doi.org/10.1101/2021.10.17.464541; this version posted October 18, 2021. The copyright holder for this preprint (which was not certified by peer review) is the author/funder, who has granted bioRxiv a license to display the preprint in perpetuity. It is made available under aCC-BY-ND 4.0 International license.

350261669 "Reversing antimicrobial resistance", DFW), and the US National Institute of

351 Child Health and Human Development (NICHD) U01HD085531 (DFW). 


\section{References}

353

1. WHO. Global tuberculosis report 2020. 2020.

2. Donald PR, Diacon AH, Lange C, Demers AM, von Groote-Biddlingmeier F, Nardell E. Droplets, dust and guinea pigs: an historical review of tuberculosis transmission research, 1878 -1940. Int J Tuberc Lung Dis 2018; 22: 972-982.

3. Van der Walt M, Moyo S. The First National TB Prevalence Survey, South Africa 2018: Short report. 2021.

4. Williams CM, Abdulwhhab M, Birring SS, De Kock E, Garton NJ, Townsend E, Pareek M, Al-Taie A, Pan J, Ganatra R, Stoltz AC, Haldar P, Barer MR. Exhaled Mycobacterium tuberculosis output and detection of subclinical disease by face-mask sampling: prospective observational studies. The Lancet Infectious Diseases 2020; 20: 607-617.

5. Houben RMGJ, Dodd PJ. The Global Burden of Latent Tuberculosis Infection: A Re-estimation Using Mathematical Modelling. PLOS Medicine 2016; 13: e1002152.

6. Auld SC, Kasmar AG, Dowdy DW, Mathema B, Gandhi NR, Churchyard GJ, Rustomjee R, Shah NS. Research Roadmap for Tuberculosis Transmission Science: Where Do We Go From Here and How Will We Know When We're There? The Journal of Infectious Diseases 2017; 216: S662-S668.

7. Wood R, Morrow C, Barry CE, 3rd, Bryden WA, Call CJ, Hickey AJ, Rodes CE, Scriba TJ, Blackburn J, Issarow C, Mulder N, Woodward J, Moosa A, Singh V, Mizrahi V, Warner DF. Real-Time Investigation of Tuberculosis Transmission: Developing the Respiratory Aerosol Sampling Chamber (RASC). PLoS One 2016; 11: e0146658.

8. Dinkele R, Gessner S, McKerry A, Leonard B, Seldon R, Koch AS, Morrow C, Gqada M, Kamariza M, Bertozzi CR, Smith B, McLoud C, Kamholz A, Bryden W, Call C, Kaplan G, Mizrahi V, Wood R, Warner DF. Capture and visualization of live Mycobacterium tuberculosis bacilli from tuberculosis patient bioaerosols. PLOS Pathogens 2021; 17: e1009262.

9. Patterson B, Morrow C, Singh V, Moosa A, Gqada M, Woodward J, Mizrahi V, Bryden W, Call C, Patel S, Warner D, Wood R. Detection of Mycobacterium 
tuberculosis bacilli in bio-aerosols from untreated TB patients Gates Open Research 2018; 1.

10. Patterson B, Bryden W, Call C, McKerry A, Leonard B, Seldon R, Gqada M, Dinkele R, Gessner S, Warner DF, Wood R. Cough-independent production of viable Mycobacterium tuberculosis in bioaerosol. Tuberculosis 2021; 126 : 102038.

11. RStudioTeam. RStudio: Integrated Development Environment for R. RStudio, PBC, Boston, MA; 2020.

12. RCoreTeam. R: A language and environment for statistical computing. $R$ Foundation for Statistical Computing, Vienna, Austria.; 2020.

13. Andrews JR, Morrow C, Walensky RP, Wood R. Integrating social contact and environmental data in evaluating tuberculosis transmission in a South African township. J Infect Dis 2014; 210: 597-603.

14. Esmail H, Dodd PJ, Houben RMGJ. Tuberculosis transmission during the subclinical period: could unrelated cough play a part? The Lancet Respiratory Medicine 2018; 6: 244-246.

15. Fennelly KP, Martyny JW, Fulton KE, Orme IM, Cave DM, Heifets LB. Coughgenerated aerosols of Mycobacterium tuberculosis: a new method to study infectiousness. Am J Respir Crit Care Med 2004; 169: 604-609.

16. Jones-Lopez EC, Acuna-Villaorduna C, Ssebidandi M, Gaeddert M, Kubiak RW, Ayakaka I, White LF, Joloba M, Okwera A, Fennelly KP. Cough Aerosols of Mycobacterium tuberculosis in the Prediction of Incident Tuberculosis Disease in Household Contacts. Clin Infect Dis 2016; 63: 10-20.

17. Theron G, Limberis J, Venter R, Smith L, Pietersen E, Esmail A, Calligaro G, te Riele J, de Kock M, van Helden P, Gumbo T, Clark TG, Fennelly K, Warren R, Dheda K. Bacterial and host determinants of cough aerosol culture positivity in patients with drug-resistant versus drug-susceptible tuberculosis. Nature Medicine 2020; 26: 1435-1443.

18. Gralton J, Tovey E, McLaws M-L, Rawlinson WD. The role of particle size in aerosolised pathogen transmission: A review. Journal of Infection 2011; 62: 113. 
414 19. Pai M, Behr MA, Dowdy D, Dheda K, Divangahi M, Boehme CC, Ginsberg A,

415 Swaminathan S, Spigelman M, Getahun H, Menzies D, Raviglione M.

416 Tuberculosis. Nature Reviews Disease Primers 2016; 2: 16076.

417 20. Patterson $B$, Wood $R$. Is cough really necessary for TB transmission?

$418 \quad$ Tuberculosis 2019; 117: 31-35.

419 21. Johnson GR, Morawska L. The mechanism of breath aerosol formation. Journal

$420 \quad$ of Aerosol Medicine and Pulmonary Drug Delivery 2009; 22: 229-237.

421 22. Haslbeck K, Schwarz K, Hohlfeld JM, Seume JR, Koch W. Submicron droplet

422 formation in the human lung. Journal of aerosol science 2010; 41: 429-438.

423 23. Edwards DA, Man JC, Brand P, Katstra JP, Sommerer K, Stone HA, Nardell E,

424 Scheuch G. Inhaling to mitigate exhaled bioaerosols. Proceedings of the

$425 \quad$ National Academy of Sciences of the United States of America 2004; 101 :

$426 \quad 17383-17388$. 
A $\begin{array}{lll}\text { i. Participant sampling ii. Real-time } & \mathrm{CO}_{2} \text { and particle detection and } & \begin{array}{c}\text { iii. Bioaerosol processing and } \\ \text { bacterial detection }\end{array}\end{array}$ Cyclone sampling, Particle sampling, $\mathrm{CO}_{2}$ sampling.
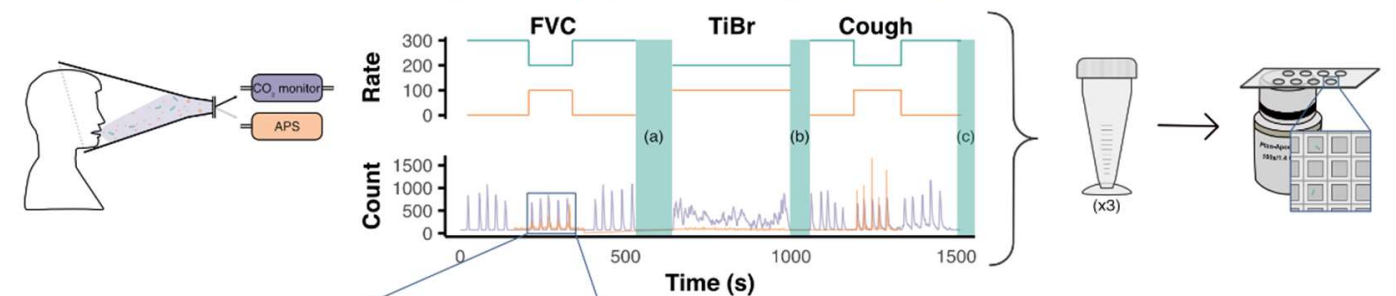

B
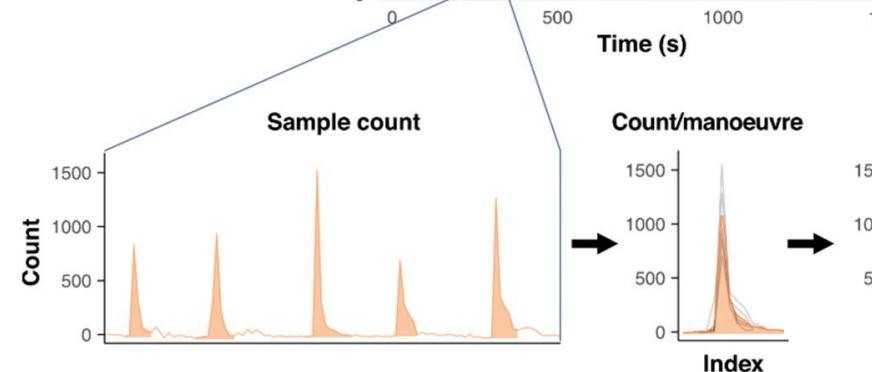

Total count 


\section{A}


\begin{tabular}{cccccc}
\hline & Level & $\boldsymbol{\beta}$ & $\mathbf{9 5 \%} \mathbf{C l}$ & Percent & $\mathbf{p}$ \\
Manoeuvre & FVC & 0.850 & $0.620,1.080$ & 607.6 & $\mathbf{8 . 5 e}-10$ \\
& Cough & 1.423 & $1.202,1.643$ & $2545.5<\mathbf{2 e - 1 6}$ \\
random effects & PTID & & & \multicolumn{3}{c}{12.5} \\
\hline
\end{tabular}

\section{C}

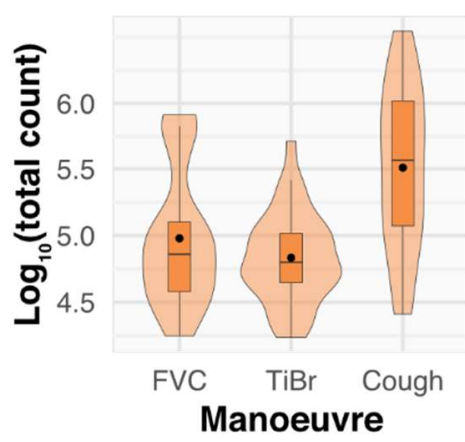

\section{Figure 2. Variation in particle production by FVC, TiBr and Cough. A}

446 comparison of the (A) sample count, (B) count/manoeuvre and (C) total count of

447 particles during sampling. The adjacent tables contain the results of univariate linear

448 mixed models for each. The beta-coefficient $(\beta)$ and 95\% confidence interval $(\mathrm{Cl})$ are

449 presented with percentage change relative to $\mathrm{TiBr}$ (Percent). The random effects

450 results indicate the degree of variation (in \%) between participants. 

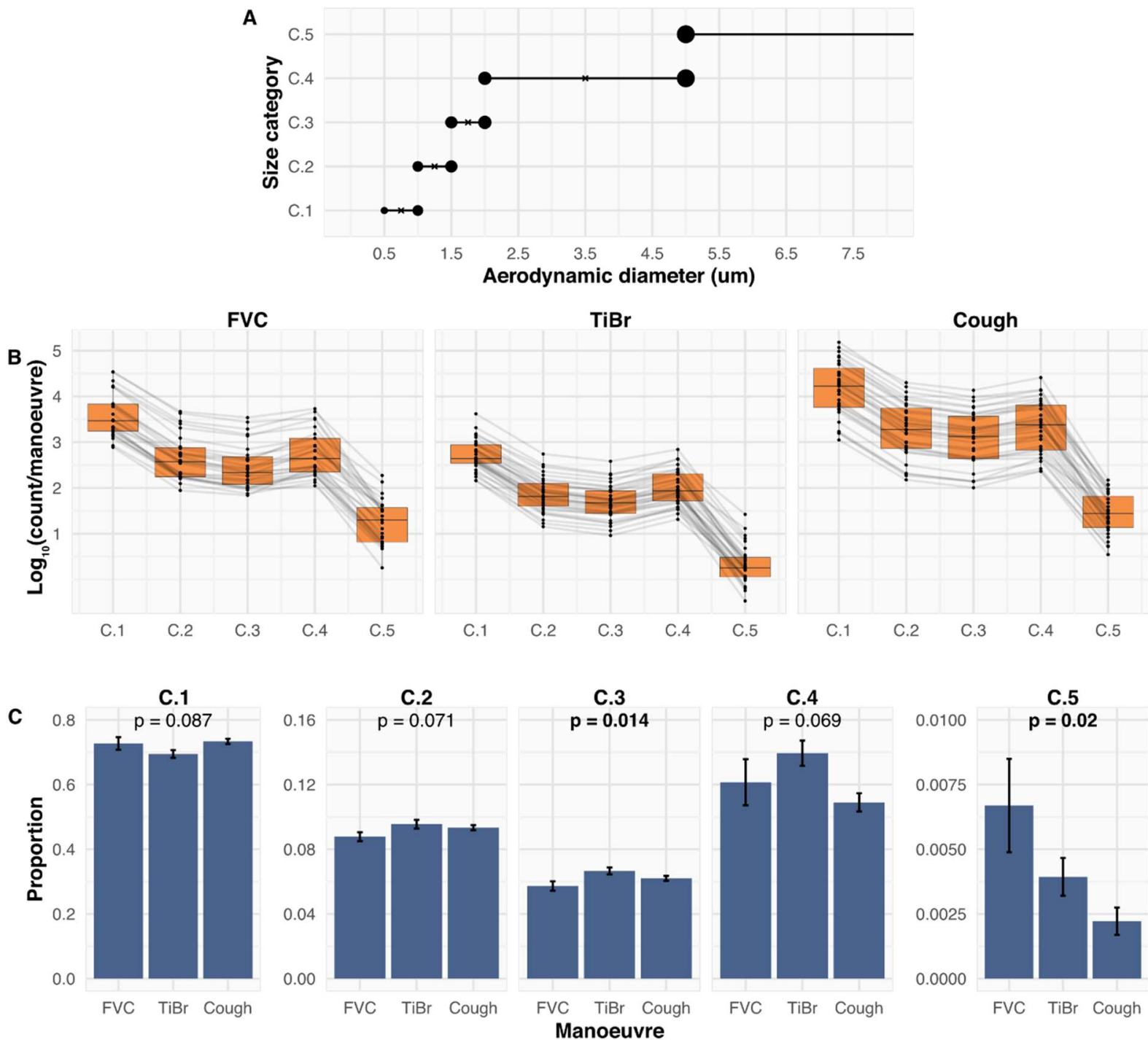

451 Figure 3. The relative contribution of particles of various sizes is consistent

452 between FVC, TiBr and Cough. (A) Graphical representation of the size categories

453 detected by the aerodynamic particle sizer. (B) A comparison of the average count

454 per manoeuvre stratified by size category. Grey lines indicate the average number of

455 particles per manoeuvre stratified by size category and participant ID (PTID). (C) A

456 comparison between manoeuvres of the proportion composition of each size

457 category per manoeuvre. The data in (C) are presented as the mean proportion \pm

458 SEM. A Repeated Measures ANOVA was performed, $p$ values below 0.05 are

459 represented in bold text. 


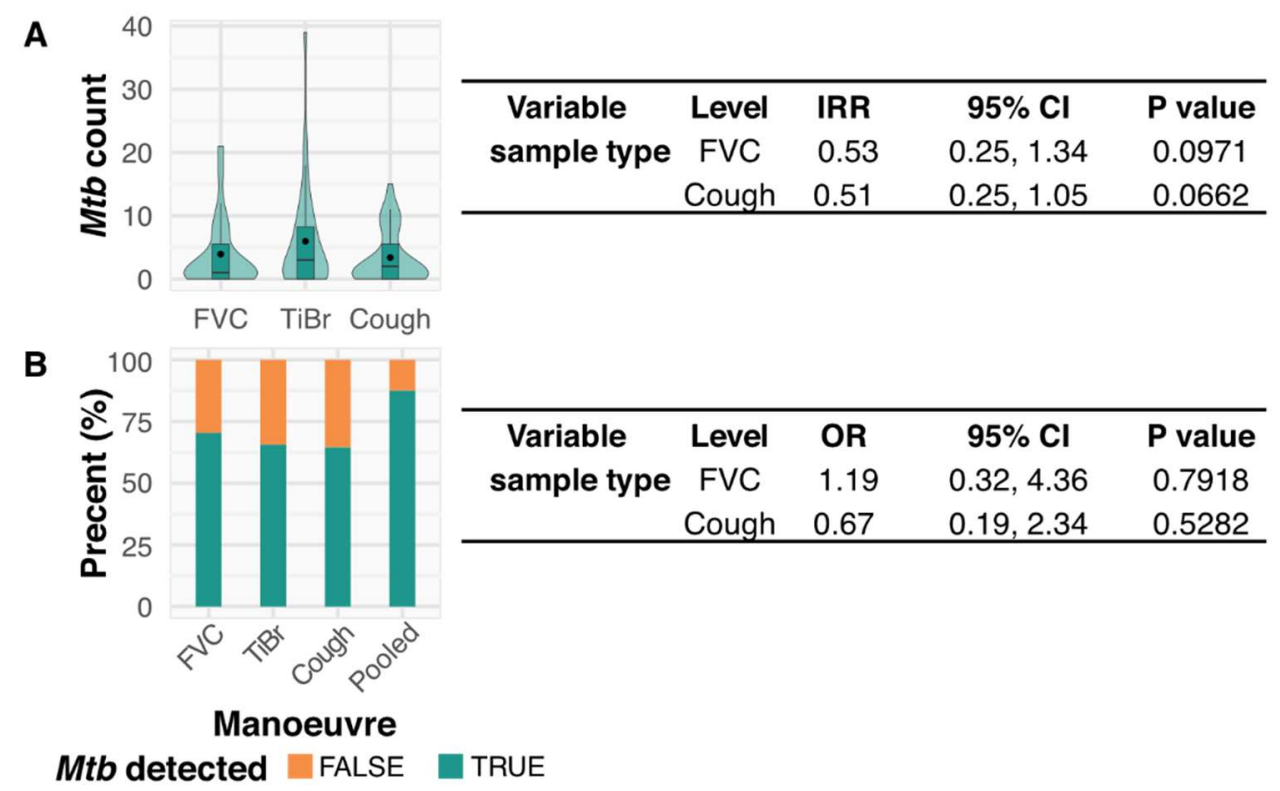

460 Figure 4. The detection of putative Mycobacterium tuberculosis within each

461 respiratory manoeuvre sample. (A) A comparison of the total number of

462 Mycobacterium tuberculosis ( $M$ tb) detected between each sample, adjacent to the

463 results from a negative binomial regression. (B) A comparison of the proportion of

464 samples that were positive for aerosolised $M t b$, adjacent to the results of a

465 generalized linear mixed model. The "pooled" variable in (B) represents the percent

466 of individuals that produced at least one positive sample. 


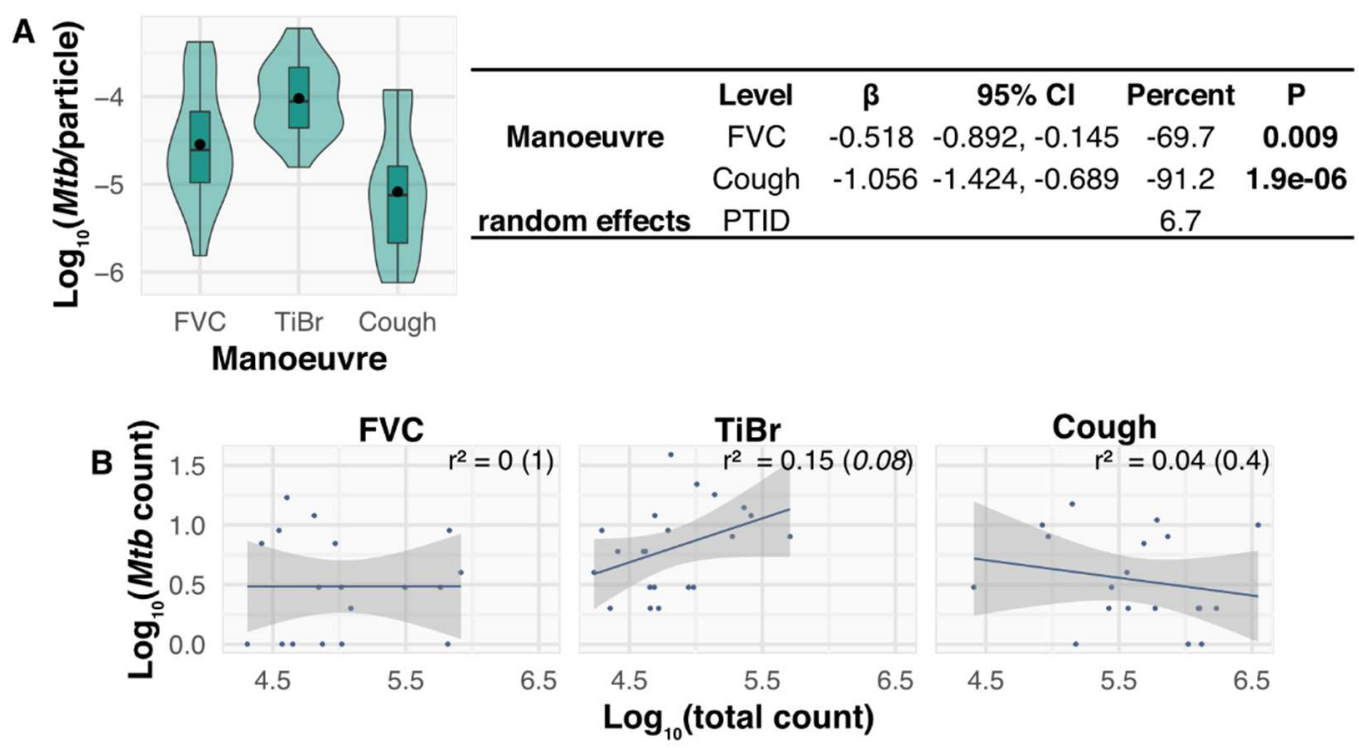

467 Figure 5. The concentration of Mycobacterium tuberculosis relative to particle

468 count for each respiratory manoeuvre. (A) A comparison of the average number

469 of Mycobacterium tuberculosis (Mtb) per particle within the bioaerosol, adjacent to

470 the results from a linear mixed model. (B) Correlation assessment between

$471 \log _{10}$ (count) and $\log _{10}(M t b)$, with the results of a Pearson's correlation ( $r$-squared $=r^{2}$

472 and $p$-value in brackets). 


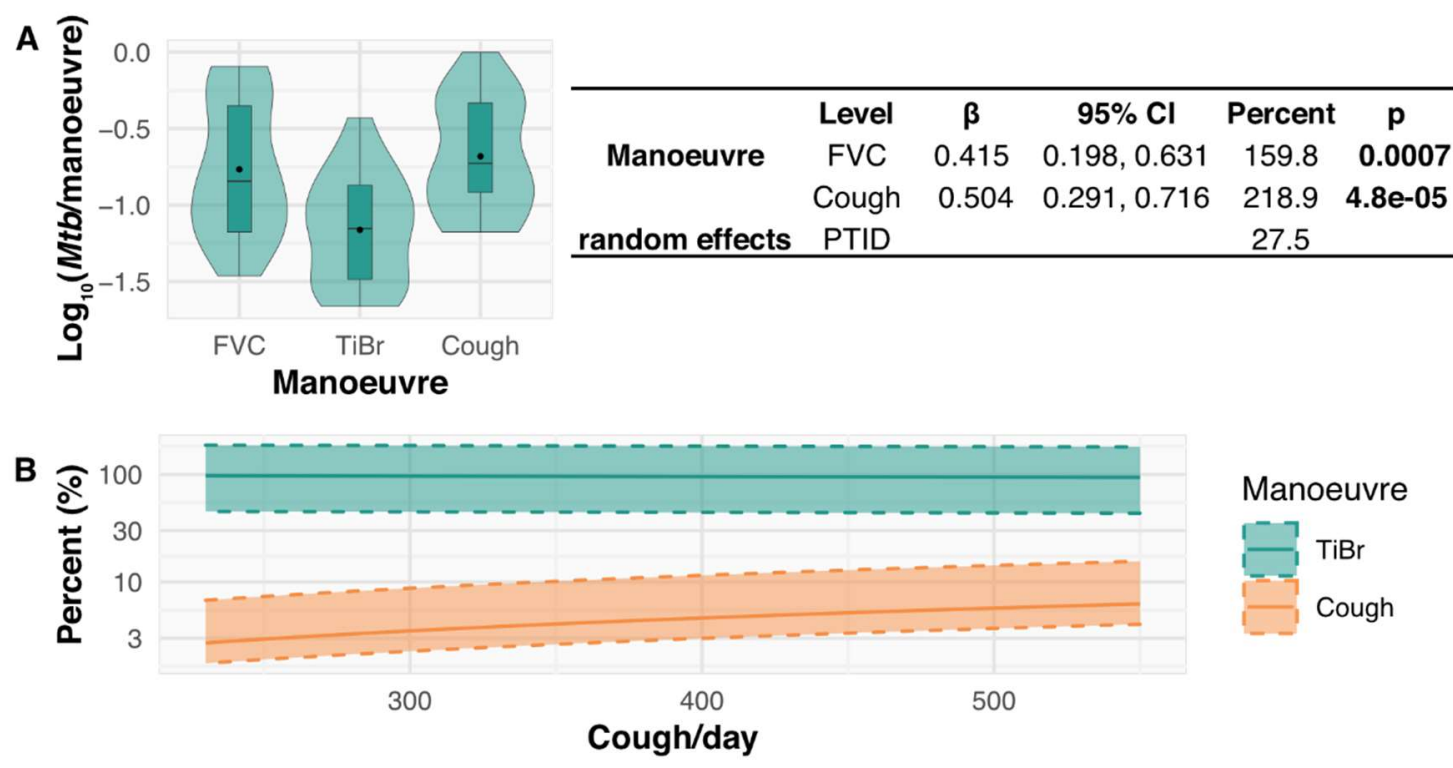

473 Figure 6. The relative contribution of Mycobacterium tuberculosis by each

474 respiratory manoeuvre. (A) A plot of the average number of Mycobacterium

475 tuberculosis (Mtb) per manoeuvre with the results of the linear mixed model. (B) The

476 relative contribution of bacteria per day (percent). We used the median frequency of

477 breaths to estimate an average of 22,047 breaths per day. We then assumed that for

478 every Cough, there would be one fewer breath throughout the day over a range of

479 coughs from $234-551$ coughs. At each cough frequency, we determined the

480 average number of bacilli per $\mathrm{TiBr}$ (solid green line) or per Cough (solid orange line).

481 We then estimated the percentage contribution of aerosolised Mtb that each

482 manoeuvre made relative to the total (solid lines sum to 100). The shaded regions

483 represent the interquartile range of $\mathrm{Mtb}$ production for both $\mathrm{TiBr}$ and Cough. 